\section{Free and forced exploration in rats as a function of between- vs within-Ss design*}

\author{
FRED P. VALLE \\ University of British Columbia, Vancouver, B.C., Canada
}

Wistar rats were given 16 open-field tests under (a) free exploration (Ss could withdraw from the field into a small dark box), (b) forced exploration, or (c) alternating free and forced exploration. Amount of locomotion and the tendency to leave the peripheral wall were initially suppressed under free exploration but recovered over tests. The tendency to rear in the field remained suppressed under free exploration over all tests. It was suggested that fear functions to inhibit locomotion under forced exploration and functions to motivate and reward escape into the small box under free exploration. As fear habituates, open-field locomotion increases.

It is of ten suggested that novel stimuli give rise to both fear and curiosity in rats. There is, however, some disagreement as to how these two hypothetical states influence locomotor activity. Valle (1971) has proposed that fear, defined as the tendency to freeze or withdraw, competes with the tendency to approach (curiosity) and that amount of forward-directed locomotor activity in an open field is determined by the difference in strength between these two tendencies. Welker (1957), however, has suggested that fear itself can produce forward-directed locomotion in an open field. Welker drew this conclusion from data that showed that rats placed in an open field from which they could not escape (forced exploration) locomoted significantly more than they did in an open field containing a small box into which they could withdraw (free exploration). Welker concluded that part of the increase in locomotion shown by rats under forced exploration reflected attempts by the animal to find a means of escape from the fear-inducing field.

Two aspects of Welker's (1957) procedure raise doubts about the generality of his interpretation, however. First, he apparently did not familiarize his Ss with the small box before the open-field tests. Thus, on free exploration trials, the rats may have been locomoting in the field less, simply because they were spending some time exploring the small box. Second, Walker used a within-Ss design: all Ss received their first three tests under free exploration conditions and the remaining tests under

\footnotetext{
*This research was supported by the National Research Council of Canada (APA 266) and the President's Committee on Research, University of British Columbia. I would like to thank Rosemary W. Bols for her assistance and Dr. G. J. Johnson for his advice concerning data analysis.
}

The open field was a square white board, $1.22 \mathrm{~m}$ on a side, with a $.3-\mathrm{m}$ wall around the periphery. The field was divided by black lines into 16 equal squares. A fine mesh screen covered the top of the field. Illumination was provided by a $100-\mathrm{W}$ white light bulb suspended $84 \mathrm{~cm}$ above the floor of the field. Illumination intensity was controlled by a transformer and was set at $0.2 \mathrm{fc}$, as measured by a Trilux footcandle meter placed on the floor of the field.

On free exploration trials, one of the walls of the field was replaced with another, which contained a 7.5-cm-wide, $12.5-\mathrm{cm}$-high doorway leading into a $20.5 \cdot \mathrm{cm}-\mathrm{long}$, 14-cm-wide, and 11-cm-high (i.d.) black box with a hinged lid. PROCEDURE

The Ss were housed in individual cages and maintained under a 7 a.m. -7 p.m. light cycle. Each day, for 10 days, the rats were handled for $3 \mathrm{~min}$ and then placed in the small box, which had been detached from the open field and placed on a small sawdust-covered table, for $3 \mathrm{~min}$. For the first minute of this period, the animals were confined to the box by a door that was then removed for the last $2 \mathrm{~min}$. Boli were removed from the box after each rat, and the box was washed with a mild vinegar-water solution at the start of each day and after every sixth rat.

Beginning on the 11 th day, the Ss were given 165 -min open-field tests, one each night between the hours of 8 p.m. and 1 a.m. On each test, the rat was placed in the corner of the field closest to where the $E$ sat while recording. On free exploration tests, the small box (with the door removed) was located one square away from this corner. One group of 12 animals was tested only under forced exploration conditions; a second group was tested only under free exploration

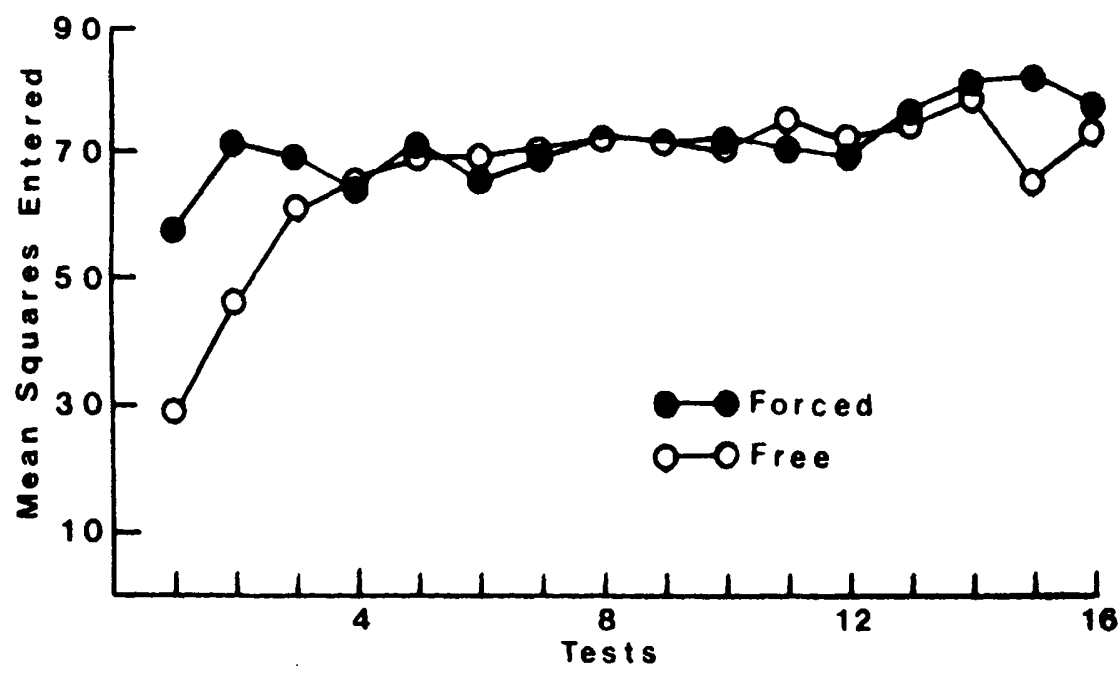

Fig. 1. Mean number of squares entered over 165 -min open-field tests by rats tested under free or forced exploration. 


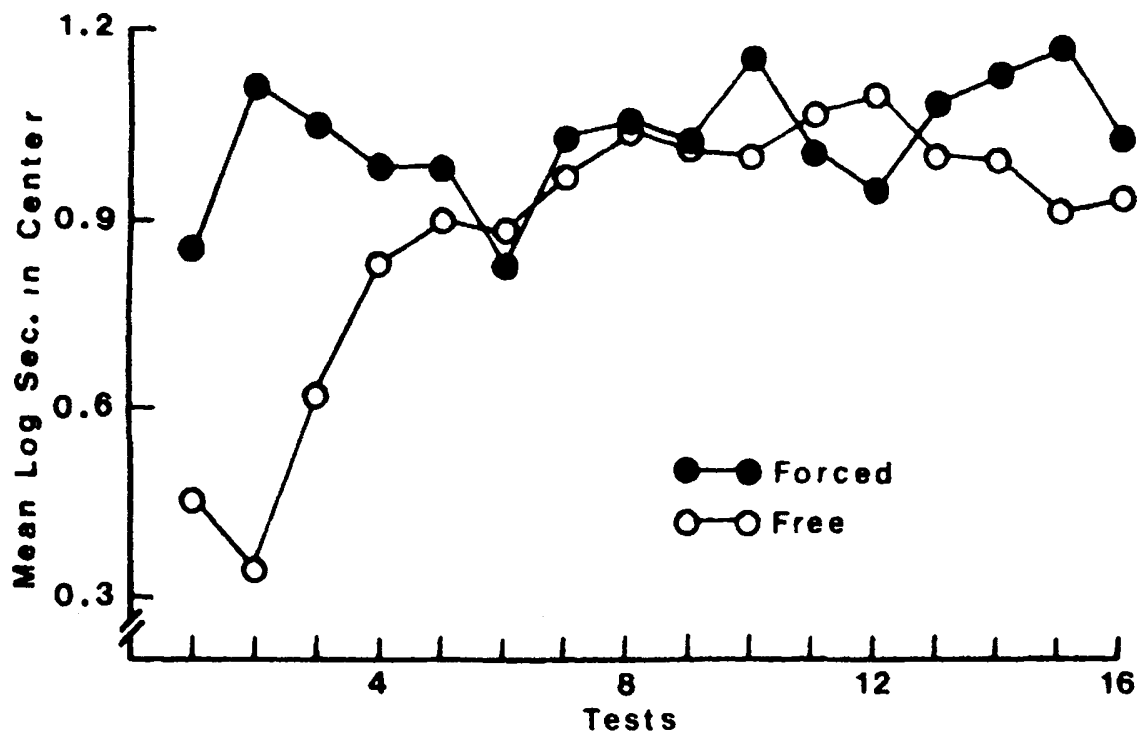

Fig. 2. Mean $\log (X+1)$ number of seconds spent in the central area of the open field by rats tested under free or forced exploration.

conditions; a third group received both forced and free exploration tests in an ABBA sequence beginning with a forced exploration test.

On all tests a record was kept of (1) the number and pattern of squares entered, with entrance defined as placing at least both forepaws in a square (complete entry into and exit from the small box also counted as entering a square), (2) the number of rears in the open field, (3) the amount of time spent in the four center squares of the open field; and (4) the number of boli (in both field and box). On free exploration tests a record was kept of the amount of time the animal spent in the open field.

After each test, the field was washed with the vinegar-water solution. The rats were maintained under ad lib food and water throughout the experiment.

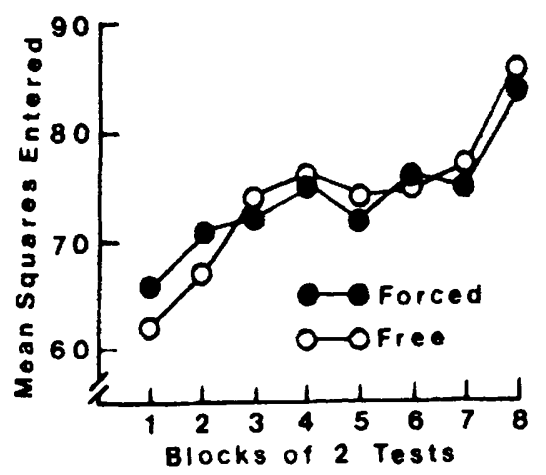

Fig. 3. Mean number of squares entered over 165 -min open-field tests by rats tested under both forced and free exploration in an ABBA sequence.
$(\mathrm{F}<1)$, and a significant interaction $(F=1.78, \mathrm{df}=15 / 330, \mathrm{p}<.05)$.

Analysis of number of boli deposited showed a nonsignificant groups effect $(F<1)$ but a significant tests effect $(F=7.61, \mathrm{df}=15 / 330$, $\mathrm{p}<.001$ ) due to the decline in defecation over tests. There was no reliable interaction. For both groups, from Test 2 on, the modal number of boli was 0 .

Inspection of time spent in the open field by the free exploration Ss as a function of tests showed a negatively accelerated increasing curve which stabilized at approximately $70 \% \cdot 75 \%$ after five tests.

\section{Within-Ss Analyses}

Figure 3 shows mean number of squares entered over blocks of two tests. It can be seen that, with the possible exception of the first two blocks of tests, the animals locomoted as much on free exploration tests as on forced exploration tests. The number of Ss (out of 12) showing more locomotion on the forced exploration half of each two-test block was as follows: $7,7,6,5,5,6,6,7$. Analysis of variance showed a nonsignificant conditions effect $(F<1)$, a significant tests effect $(F=5.35, \mathrm{df}=7 / 77$, $\mathrm{p}<.001)$, and a nonsignificant interaction $(F<1)$.

Figure 4 shows mean $\log (\mathrm{X}+1)$ number of seconds spent in the central area of the field. The Ss spent more time in the center on forced exploration tests than on free exploration tests, with the difference being most pronounced on the first four blocks of tests. Analysis of variance indicated a marginal conditions effect $(\mathrm{F}=3.74, \mathrm{df}=1 / 11$, $.05<p<.10)$ and nonsignificant tests $(F=1.51, \mathrm{df}=7 / 77)$ and interaction $(\mathrm{F}=1.36, \mathrm{df}=7 / 77)$ effects. Analysis of thigmotactic ratios failed to show any significant effects.

There was substantially more

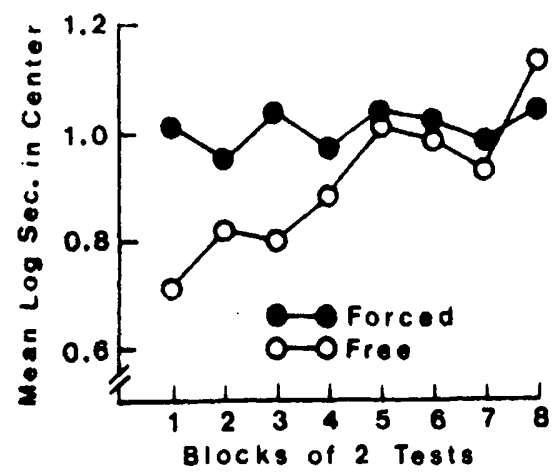

Fig. 4. Mean $\log (X+1)$ number of seconds spent in the central area of the open field by rats tested under both forced and free exploration in an ABBA sequence. 
rearing in the field on forced exploration tests than on free exploration tests throughout the series, although rearing declined somewhat over tests under forced exploration and increased somewhat under free exploration. The number of animals showing more open-field rearing on the forced exploration half of each two-test block was as follows: $12,10,12,12,10,10,12,9$. Analysis of variance showed a significant conditions effect $(F=303.66$, $\mathrm{df}=$ $1 / 11, p<.001)$, a nonsignificant tests effect $(F=1.64$, df $=7 / 77)$, and a significant interaction $(\mathrm{F}=3.26, \mathrm{df}=$ $7 / 77, \mathrm{p}<.01)$.

Frequency of defecation was higher on forced exploration tests than on free exploration tests, al though, by the third block of tests, the modal number of boli on both types of tests was 0 . Analysis of variance showed a significant conditions effect $(F=9.15$, $\mathrm{df}=1 / 11, \mathrm{p}<.05)$, a significant tests effect $(F=4.36, \mathrm{df}=7 / 77, p<.01)$, and a nonsignificant interaction $(\mathrm{F}<1)$.

\section{Comparisons of Between} vs Within -Ss Data

For all response measures, the data from "within" Ss were compared with data obtained on corresponding trials from "between" sis. The only reliable differences obtained all involved data from free exploration tests. "Within" Ss spent more time in the central area of the field on the early free exploration tests than did "between" Ss, as shown by a reliable interaction between groups and tests $(F=2.59, \mathrm{df}$ $=7 / 154, p<.05)$. "Within" Ss also had higher thigmotactic ratios than "between" Ss on the early free exploration tests, as shown by the Groups by Tests interaction $(F=3.05$, $\mathrm{df}=7 / 154, \mathrm{p}<.01)$. With regard to amount of locomotion, although the Groups by Tests interaction failed to reach significance, "within" Ss did locomote reliably more than "between" Ss on Test 2 (the first free exploration test for the "within" Ss). "Within" Ss reared more often on free exploration tests than did "between" Ss, as shown by a reliable groups effect $(\mathrm{F}=5.50, \mathrm{df}=1 / 22, \mathrm{p}<.05)$. And "within" Ss defecated more on the early free exploration tests than did "between" Ss, as shown by a reliable Groups by $\mathrm{Tes}$ ts interaction $(\mathrm{F}=2.81$, $\mathrm{df}=7 / 154, \mathrm{p}<.05)$. It should also be noted that the comparison of "within" and "between" Ss on forced exploration tests showed a marginal groups effect for frequency of defecation $(\mathrm{F}=3.62, \mathrm{df}=1 / 22$, $.05<\mathrm{p}<.10)$, suggesting that "within" Ss defecated more than did "between" Ss on both free and forced exploration tests.

\section{DISCUSSION}

The results of the present experiment show that, initially, rats tested under forced exploration locomote more than rats tested under free exploration. Since the free exploration Ss were familiar with the small box, it seems unlikely that this difference in open-field activity could be due to exploration of the small box by free exploration Ss. Rather, it seems reasonable to conclude that the open field gave rise to fear and that escape into the small box was rewarded by fear reduction. Thus, on the initial tests, the open-field activity of free exploration Ss was suppressed by response competition. After three to four tests, the open-field locomotion of free exploration Ss increased until it equaled that of forced exploration Ss. Again, it seems reasonable to conclude that this increase was due to habituation of fear in the open field: withdrawal into the small box was no longer as rewarding. To some extent then, the difference in open-field locomotion between forced and free exploration $S s$ is probably due to two factors: (1) under free exploration, withdrawal into the small box is rewarded by a substantial reduction in fear and hence withdrawal effectively competes with open-field locomotion; (2) withdrawal into the small box delays habituation to the fear-inducing stimuli of the field and hence the effects of fear are prolonged in the free exploration animals.

If it is granted that the effect of fear on free exploration is to suppress open-field locomotion because of the rewarding effects of escape into the small box, what then of the effects of fear on open-field locomotion under forced exploration? Welker (1957) proposed that fear facilitated or activated open-field locomotion under forced exploration, while Valle (1971) proposed that fear inhibited open-field locomotion. As can be seen in Fig. 1, the locomotion scores of forced exploration Ss increased from Test 1 to Test 2 and remained relatively constant thereafter. This is the pattern to be expected if (1) fear inhibits locomotion and (2) a substantial amount of habituation occurs on the first forced exploration test.

That a single forced exposure to the field can produce a marked reduction in fear is suggested by the fact that on Test 2, "within" Ss (who received a forced exploration experience on the first test) locomoted significantly more under free conditions than did "between" Ss (who received a free exploration experience on the first test). ${ }^{1}$ Thus, the data of the present experiment are consistent with the hypothesis that fear inhibits the locomotor activity of animals forced to remain in the fear-inducing situation and that as fear habituates, locomotion may increase (as long as curiosity does not habituate at a faster rate than fear-see Valle, 1971).

There was also a substantial and permanent decrement in frequency of rearing under forced exploration. Part of this decrement may have been due to the fact that some rearing occurred in the small box on free exploration tests and this activity was not recorded. It is not clear, however, why open-field rearing remained suppressed under free exploration while open-field locomotion became completely disinhibited. Since "within" Ss reared significantly more often than "between" Ss on free exploration tests, it appears again that the behavioral effects of the free exploration situation can be modified by forced exploration experience.

Frequency of defecation was differentially affected by the type of design used. While there was no free vs forced effect in the between-Ss analysis, such an effect appeared in the within-Ss analysis. In part, this pattern may simply reflect the greater sensitivity of the within-Ss design. Sensitivity of design cannot be the whole explanation, however, since "within" Ss defecated more on corresponding trials than both free exploration and forced exploration "between" Ss. The results of the present experiment indicate, therefore, that while alternating between free and forced exploration produces more open-field locomotion and rearing (on free exploration tests) than does free exploration alone, alternating between the two produces more defecation than does free exploration alone.

\section{REFERENCES}

VALLE, F. P. Rats' performance on repeated tests in the open field as a function of age. Psychonomic Science, $1971,23,333-335$.

WELKER, W. 1. "Free" versus "forced" exploration of a novel situation by rats. Psychological Reports, 1957, 3, 95-108. NOTE

1. This difference was not due to the possibility that "within" Ss might have taken longer to find the small box. Both groups entered a similar number of open-field squares before entering the small box for the first time on that test, the means being 2.7 and 3.2 for the "between" and "within" Ss, respectively. 